\section{T. Batchelor}

\section{Lymphoma of the Nervous System}

Butterworth Heinemann Health, 2004

207 pp.; GBP 40.99

ISBN 0-7506-7406-7

PPrimary central nervous system lymphoma (PCNSL) was once a rare brain tumour subgroup and only occasionally mentioned in case reports; however, in recent years, it has gained more interest in experimental and clinical medicine, as nowadays, a new PCNSL is being diagnosed in about 1,000 people each year in the United States. During the last years, chemotherapy has become the standard in adjuvant therapy; its results seem to be imperfect but superior to radiation alone among the many studies reported.

The book is subdivided into three main sections with overall 14 chapters, of which each is written by an opinion leader of the field from the United States. After an introductory chapter with basic information on lymphomas, specifically written for non-oncologists, the authors describe the current experimental and clinical knowledge in pathology, epidemiology, neuroimaging and treatment of both AIDS and non-AIDS-associated PCNSL in the first section. The text is clearly focused on clinically relevant aspects and is underlined by excellent tables, figures and diagrams. Especially, the chapter dealing with neuroimaging is of outstanding interest as the state-of-the-art-reflecting methods in imaging PCNSL are presented and discussed in detail; even magnetic resonance spectroscopic imaging that is not routinely used at present, but represents a promising method for the future, is described in some aspects. Besides its increasing diagnostic value, neuroimaging of PCNSL will play a leading role to further improve the treatment of patients with PCNSL in the near future: imaging may reveal the assessment of the 'location', 'magnitude', and 'duration' of the cellular answer to therapeutic options and visualize the relation to the clinical therapeutic effect. In addition, neuroimaging may help to substantially improve the diagnostic accuracy (e.g., brain biopsy) or may completely change the diagnostic procedure of brain tumours in the near future. Such specification of current research data is missing; however, this does not impair the excellent impression of this book. These first chapters also include the latest information on the use of radiation and chemotherapy for PCNSL and other types of lymphoma; the neurotoxicity of such therapeutic procedure is mentioned.

In the second section, the unique neurological complications of lymphoid malignancies are described. This focus on relatively rarely described, but clinically important sings and symptoms is discussed spanning basic science, diagnostic criteria, and therapeutic options, well demonstrating that - in the case of secondary central nervous system lymphoma - the periphery nervous system can be involved in the disease as well. In this section, the few international groups whose research is focused on this feature are well cited, so that interested readers can deepen their knowledge of these specific aspects. The summary tables are well suited for those among the readers that are not familiar with the demanding diagnostic procedure of such syndromes.

The last and third section sheds light on some specific aspects of central nervous system lymphoma. This section is of interest, even though the choice of chapters appears somewhat random: one would, for example, expect the aspects of brainstem lymphoma to be included. The chapter on neurolymphomatosis is well written, though it is clear that such a complex theme cannot be debated in detail in one single chapter.

All in all, the book is highly recommendable for all clinical and research neuroscientists and oncologists that are interested in this 'fascinating' and treatment-sensitive brain tumour subgroup. It not only provides practical guidance on the recognition and treatment of lymphoma of the nervous system, but also sheds light on potential future research areas.

Bernhard Schaller, Stockholm 